\title{
NUCLEAR DATA UNCERTAINTY QUANTIFICATION FOR THE DECAY HEAT OF PWR MOX FUELS USING DATA ASSIMILATION OF ELEMENTARY FISSION BURSTS
}

\author{
V. Vallet ${ }^{1}$, J. Huyghe ${ }^{1}$, C. Vaglio-Gaudard ${ }^{1}$, D. Lecarpentier ${ }^{2}$, and C. Reynard-Carette ${ }^{3}$ \\ ${ }^{1}$ CEA, DEN, DER Cadarache \\ F-13108 Saint Paul-lez-Durance, France \\ ${ }^{2}$ EDF Research and Development \\ 7 Bd Gaspard Monge, 91120 Palaiseau, France \\ ${ }^{3}$ Aix Marseille Université, Université de Toulon, CNRS, IM2NP \\ Marseille, France \\ vanessa.vallet@ecea.fr
}

\begin{abstract}
Currently there is no integral experimental data for code validation regarding the decay heat of MOX fuels, excepted fission burst experiments (for fission products contributions at short cooling times) or post-irradiated experiments on nuclide inventories (restricted number of nuclide of interest for decay heat). The uncertainty quantification mainly relies on uncertainty propagation of nuclear data covariances. In the recent years, the transposition method, based on the data assimilation theory, was used in order to transpose the experimentto-calculation discrepancies at a given set of parameters (cooling time, fuel burnup) to another set of parameters. As an example, this method was used on the CLAB experiments and the experiment-to-calculation discrepancies at 13 years were transposed to an UOX fuel between 5 and 27 years and for burnups from 10 to $50 \mathrm{GWd} / \mathrm{t}$. The purpose of this paper is to study to what extent the transposition method could be used for MOX fuels. In particular, the Dickens fission burst experiment of ${ }^{239} \mathrm{Pu}$ was considered for MOX fuels at short cooling times $(<1 \mathrm{~h} 30)$ and low burnup $(<10 \mathrm{GWd} / \mathrm{t})$. The impact of fission yields $(\mathrm{FY})$ correlations was also discussed. As a conclusion, the efficiency of the transposition process is limited by the experimental uncertainties larger than nuclear data uncertainties, and by the fact that fission burst experiments would only be representative to the FY contribution of the decay heat uncertainty of an irradiated reactor fuel. Nevertheless, this method strengthens the decay heat uncertainties at very short cooling times, previously based only on nuclear data covariance propagation through computation.
\end{abstract}

KEYWORDS: Decay heat, MOX fuels, uncertainty propagation, transposition, data assimilation

\section{INTRODUCTION}

The decay heat (DH) is a crucial issue for safety design and operation in both normal and accidental scenarios, from reactor shutdown until geological times. At the CEA, decay heat computations are performed with the DARWIN2.3 package [1]. The DARWIN2.3 package is the French reference tool for spent nuclear fuel characterization, based on the JEFF-3.1.1 library [2]. The confidence and accuracy of 
decay heat computations are ensured by the Verification, Validation and Uncertainty Quantification process (VV\&UQ) of the DARWIN2.3 package.

For the validation part of the VV\&UQ process, DARWIN2.3 benefits from a large experimental database [1]: Post Irradiated Experiments for the validation of the spent fuel inventory, calorimetric measurements of fuel rods/assemblies for the validation of the decay heat of UOX fuels (MERCI-1 experiment, CLAB) and Fission Burst Experiments (FBE). The FBE consist in the measurement of the heat emitted after the fission of a small amount of matter containing a fissile nuclide at a given neutron incident energy. Several FBE have been performed for the ${ }^{239} \mathrm{Pu}_{\mathrm{th}}$ in the $80 \mathrm{~s}$ for times ranging from 1 second to 1 day with experimental uncertainty under $10 \%$ at one standard deviation (std) [3] [4] [5].

The problem is that there is currently no integral data available such as calorimetric measurements that would allow an experimental validation of the decay heat computations of PWR MOX fuels. The experimental validation of MOX fuels mainly relies on the exploitation of FBE and/or assay data.

At short cooling times, the decay heat is mainly driven by radioactive decay of fission products. After one hour, $80 \%$ of the total decay heat comes from the fission products. After one day it is still $70 \%$ of the total decay heat. In addition to that, in a MOX fuel, about $70 \%$ of the fission events are due to the thermal fission of ${ }^{239} \mathrm{Pu}$. These are the underlying motivations to look at the transposition method and to assess whether or not the FBE of ${ }^{239} \mathrm{Pu}$ could be used in order to help quantifying biases and uncertainties due to nuclear data for MOX fuels decay heat computations.

The transposition method has already been used with the CLAB set of data to the decay heat of PWR UOX fuels [6]. The goal was to quantify to what extent a measurement at a given set of parameters

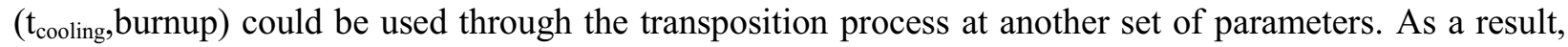
the transposition of the calculation/experiment $(\mathrm{C} / \mathrm{E})$ discrepancies of the CLAB assemblies can be done for PWR UOX fuels at cooling times from 5 to 27 years and for burnups from 10 to $50 \mathrm{GWd} / \mathrm{t}$ at least.

This method has also been applied to PWR UOX fuels with the MERCI-1 experiment [7]. This experiment consisted in the calorimetric measurement of the decay heat of a fresh UOX fuel $\left(3.7 \%{ }^{235} \mathrm{U}\right)$ irradiated up to $3.6 \mathrm{GWd} / \mathrm{t}$ between 45 minutes and 42 days of cooling. The study demonstrated that the MERCI-1 experiment is representative to UOX fuels up to $10 \mathrm{GWd} / \mathrm{t}$ between 5 minutes and 50 days of cooling. The transposition method, based on the data assimilation theory, can also provide feedback on nuclear data, as it was done in [8], again with the MERCI-1 experiment.

\section{PRESENTATION OF THE DARWIN2.3 PACKAGE}

\subsection{Description of the DARWIN2.3 package for PWR calculations}

In the PWR calculation route, the DARWIN2.3 package [9] involves APOLLO2, a 2D lattice neutron code, and PEPIN2, a depletion code, whose strength is to have almost complete filiation chains, describing more than 3800 radionuclides. The neutron code APOLLO2 is used in order to produce an input data file for PEPIN2. This file gathers both multigroup self-shielded cross-sections and neutron spectra as a function of the burnup, for the studied fuel. The PEPIN2 depletion code uses the input data file provided by APOLLO2 in order to produce a collapsed library with burnup dependent cross-sections. This library is completed with cross-sections from JEFF-3.1.1 for the missing isotopes in the APOLLO2 filiation chains, and completed with decay data and fission yields (FY) data coming from the JEFF-3.1.1 library as well. A very precise depletion history can be given to the PEPIN2 code, with intra-cycles for instance, power variations and cooling periods. 


\subsection{Description of the sensitivity and uncertainty propagation method}

Recently, a deterministic uncertainty propagation method has been implemented in the DARWIN2.3 package. This method is based on a Taylor series expansion at first order of the integral quantity of interest $(\mathrm{Y})$. The variance of a linear function of uncertain parameters can be written in a matrix from according to the formula (1), better known as the "sandwich rule".

$$
\operatorname{var}(Y)=\sqrt{S_{Y / D}^{T} D S_{Y / D}}
$$

In this formulation, $\mathrm{D}$ is the covariance matrix of the uncertain parameters, i.e. the nuclear data (ND) and $\mathrm{S}_{\mathrm{Y} / \mathrm{D}}$ is the sensitivity matrix of $\mathrm{Y}$ to ND. The ND uncertainties taken into account are the decay data (mean decay energies, decay periods and branching ratios), the independent FY and the 1-group crosssections and their isomeric ratios. This method relies on the hypothesis of linearity of Y (here the decay heat) to the uncertain parameters (here the ND). The linearity hypothesis has been checked on several cases that allowed the use of this method for PWR decay heat computations [10]. The sensitivity profiles are evaluated by direct perturbation of the uncertain input parameters.

\subsection{Description of the nuclear data libraries and associated covariances}

The nuclear data libraries used within the DARWIN2.3 package are derived from the JEFF-3.1.1 European evaluation [2]. The associated uncertainties are also read in the JEFF-3.1.1 library for independent FY and decay data. The OECD/NEA Working Party on International Nuclear Data Evaluation Cooperation (WPEC), subgroup 37, on improved fission product yield evaluation methodologies, provided four sets of covariance matrices, all consistent with the JEFF-3.1.1 library [11], [12], [13], [14]. These covariances mainly result in a better description of the modeling and physical constraints between independent FY. The use of these matrices results in a strong reduction of the decay heat uncertainty due to nuclear data. In this paper, the impact of the CEA set of covariance data [11] in the decay heat uncertainties and on the representativity calculations will be quantified.

The covariance matrices associated with neutron cross-sections are taken from the COMAC database [15]. This database provides, among other things, covariance matrices in a multigroup format (26 or 33 groups) for the main radionuclides involved in reactor physics and back-end cycle. Differential and integral experiments are used in the re-estimation process of nuclear data and to derive reliable covariance matrices for the main actinides in the COMAC database [16], [17].

\subsection{Description of the experimental validation of the Dickens fission burst experiment}

In this paper we focused on the Dickens [18] experiments. This set of data covers a large range of cooling times, from 3 seconds to 12000 seconds ( $\sim 3.3$ hours). The total energy-release rate was deduced from the separate measurement of the $\beta$ and $\gamma$ contributions with beta and gamma spectrometers. For the ${ }^{239} \mathrm{Pu}$ experiment, small samples of 1 and $5 \mu \mathrm{g}$ of plutonium oxide $\left(99 \%{ }^{239} \mathrm{Pu}\right)$ were submitted under a thermal neutron flux in the Oak Ridge Research Reactor during 1, 5 and 100 seconds of irradiation. A strong effort has been made on determining experimental covariance matrix. The uncertainties taken into account are related to the determination of the total number of fission, the detectors, the fission-gas loss, etc. The global uncertainties are comprised between 2 and $5 \%$ at one standard deviation (std).

The experimental values (E) are compared to the calculated ones (C) with the DARWIN2.3 package (see Fig. 1). The calculation is in agreement with the experimental data, the $\mathrm{C}$ and $\mathrm{E}$ values overlap each other within the error bars at 2 std. The C/E-1 discrepancies are comprised between $-7 \%$ and $+1 \%$. The error bars associated with the calculated values are the uncertainties due to ND covariances, propagated 
through the decay heat calculation, comprised between 2 and $4 \%$ at one std. Experimental and ND uncertainties are of the same order of magnitude.

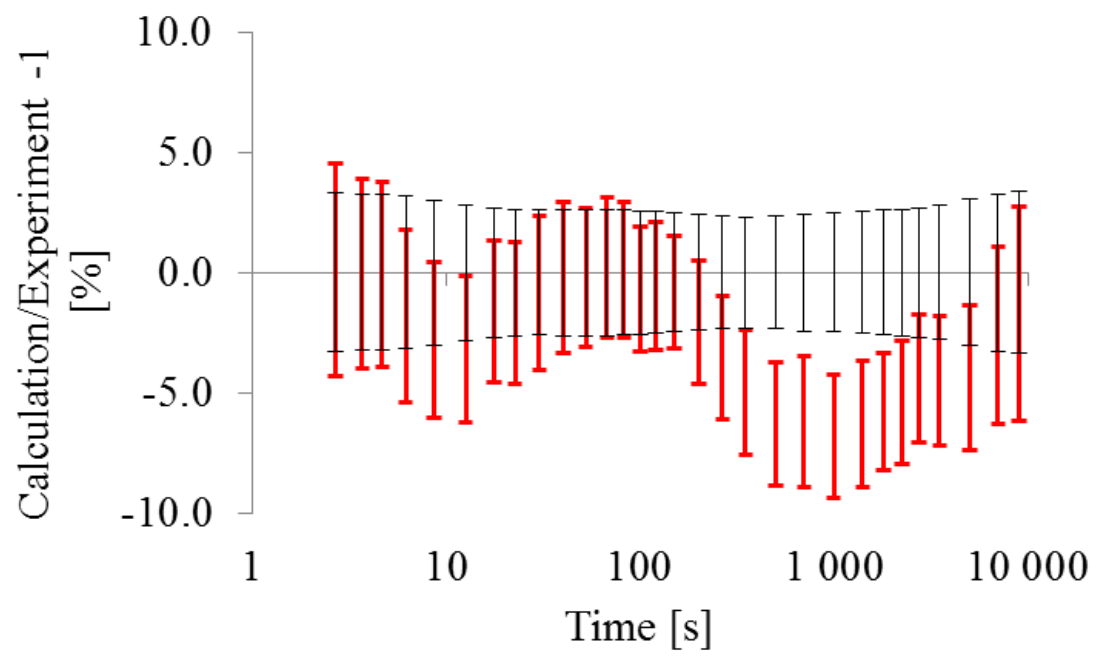

$\mathrm{C} / \mathrm{E}-1+\exp$.

Unc. (1 std)

Nuclear data

unc. (1 std)

Figure 1. C/E discrepancies between DARWIN2.3 and the Dickens ${ }^{239}$ Pu FBE.

\section{DESCRIPTION OF THE REPRESENTATIVITY/TRANSPOSITION METHOD}

The representativity and transposition method relies on an experimental data assimilation process [17]. The transposition is possible when the similarity between the experiment and the "reactor" case is strong. This similarity is quantified by the representativity factor, introduced by Orlov [19]. The representativity factor is a correlation factor between an experiment and the "reactor" case regarding ND uncertainty for a physical parameter (decay heat in this case). Let $\mathrm{R}$ and $\mathrm{E}$ be the subscripts referring to a reactor and an experiment. Let $\mathrm{Y}$ be the calculation of an integral quantity (the decay heat here). $\mathrm{S}_{\mathrm{R}}$ and $\mathrm{S}_{\mathrm{E}}$ are the sensitivity profiles of $\mathrm{Y}$ to ND for the reactor case and for the experiment. Then, the representativity factor is given by the formula (2). $\mathrm{r}_{\mathrm{R}, \mathrm{E}}$ represents the share of information provided by the experiment $\mathrm{E}$ with the $\mathrm{R}$ application regarding the $\mathrm{Y}$ quantity. Both $\mathrm{r}_{\mathrm{R}, \mathrm{E}}$ and a priori uncertainties strongly depend on the quality of the covariance matrices.

$$
r_{R, E}=\frac{S_{R}^{T} D S_{E}}{\sqrt{S_{R}^{T} D S_{R}} \sqrt{S_{E}^{T} D S_{E}}}=\frac{S_{R}^{T} D S_{E}}{I_{R} I_{E}}
$$

One can also define a weight $w$ (see formula (3)) where $\sigma_{e}$ is the uncertainty associated with the experiment and $I_{E}$ the prior uncertainty of the experiment due to ND. The weight $w$ provides an indication of the interest of the experiment regarding a transposition application. In the ideal case where there is no experimental uncertainty $\left(\sigma_{E}=0\right)$, the weight is maximum and the main source of uncertainty comes from the ND. As a conclusion, the transposition method is the most efficient, that is to say it leads to the largest bias and uncertainty reduction due to ND covariances, when $r_{R, E}$ and $w$ are the closest to unity.

$$
w=\frac{1}{1+\sigma_{E} / I_{E}}
$$

The transposition method applied to one experiment and one reactor application allows an indirect re-estimation of nuclear data leading to a new calculation bias $\left(\delta Y^{*}\right)$ and a posterior uncertainty $\left(I_{R}^{*}\right)$ due 
to ND covariances (see formulas (4) and (5)) [20]. To apply this method, the modeling calculation biases must be limited.

$$
\begin{gathered}
\delta Y^{*}=\frac{Y_{R}^{*}-Y_{R}}{Y_{R}}=\frac{r_{R, E}}{1+\left(\frac{\sigma_{E}}{I_{E}}\right)^{2}} \frac{I_{R}}{I_{E}}\left(\frac{E-Y_{E}}{Y_{E}}\right)=r_{R, E} W \frac{I_{R}}{I_{E}}\left(\frac{E-Y_{E}}{Y_{E}}\right) \\
\frac{I_{R}^{*}}{I_{R}}=\sqrt{1-w r_{R, E}^{2}}
\end{gathered}
$$

\section{APPLICATION TO A MOX FUEL}

This paper focuses on a MOX fuel with a $5.4 \%$ plutonium content $\left(1.2 \%{ }^{238} \mathrm{Pu}, 59.7 \%{ }^{239} \mathrm{Pu}, 24.1 \%{ }^{240} \mathrm{Pu}\right.$, $8.4 \%{ }^{241} \mathrm{Pu}, 4.7 \%{ }^{242} \mathrm{Pu}$ and $\left.1.9 \%{ }^{241} \mathrm{Am}\right)$. The study is restricted to low burnups (500 MWd/t, 1000 $\mathrm{MWd} / \mathrm{t}$ and $10 \mathrm{GWd} / \mathrm{t}$ ). At higher burnups, the independent $\mathrm{FY}$ of ${ }^{241} \mathrm{Pu}$ has a stronger influence in the decay heat uncertainty. The cooling times considered for the MOX fuel vary from 100 seconds to 5000 seconds $(\sim 1 \mathrm{~h} 30)$. Under 100 seconds of cooling, the total decay heat is mainly driven by delayed neutrons. The Dickens FBE provides total (beta and gamma) DH until 12000 seconds.

All the results are summarized in Table I (with FY correlated) and Table II (with FY uncorrelated). These tables provide all the ingredients required to do the transposition of the Dickens $\mathrm{C} / \mathrm{E}$ discrepancies on the decay heat computations of MOX fuels. To begin with, the comparison of Table I and Table II ( $\mathrm{I}_{R}$ column) shows the impact of taking into account the fission yields correlations in the uncertainty propagation of ND covariances. At these short cooling times, FY correlations do not change the ND uncertainty on the decay heat. This is not the case at longer cooling times. The variance decomposition of the DH uncertainty of MOX fuels is mainly driven by the FY contribution $\left(\sim 65 \% \mathrm{FY}{ }^{239} \mathrm{Pu}_{\mathrm{th}}, \sim 6 \% \mathrm{FY}\right.$ ${ }^{241} \mathrm{Pu}_{\text {th }}$ and $\left.\sim 2 \% \mathrm{FY}{ }^{238} \mathrm{U}_{\text {fast }}\right)$ and the cross sections contribution $(\sim 20 \%)$ and to a lesser extent to decay energies $(\sim 7 \%)$.

For each cooling time of interest for the MOX fuel (from 100 seconds to 5000 seconds), the representativity factor is computed for all the Dickens times. The optimum is often obtained for $t_{\text {DICKENS }}$ longer than $\mathrm{t}_{\mathrm{MOX}}$. Indeed, for a fission burst, there is no depletion period before cooling: the fission products distribution is equal to the FY distribution of the fissile nuclide considered. On the contrary, for a MOX fuel, the depletion period has a strong impact on the distribution of fission products at the beginning of the cooling period. During the irradiation, short-life fission products have already decayed into long-life daughters. Without (resp. with) FY correlations, the $r_{R, E}$ factor is about 0.7 (resp. 0.8). These values are below the threshold of 0.9 usually considered to assess an effective transposition, that is to say with a strong ND uncertainty reduction [20]. Nevertheless, this value is consistent with the fact that the FBE is only sensitive to ${ }^{239} \mathrm{Pu} \mathrm{FY}$ and associated decay data. These ND count for about $70 \%$ of the MOX variance. Moreover, the $w$ value (see formula (3)) is about 0.5 because the experimental uncertainties and the nuclear data uncertainties associated with the FBE are close. With $w=0.5$ and $r_{R, E}=0.7$, the uncertainty reduction is about $13 \%$ according to formula (5), and the bias transfer would be about 0.2 , with large E/C-1 discrepancies. This is not the ideal situation to have an effective transposition of the experimental information.

The results of the transposition process are summarized in the last two columns of Table I and Table II: $\delta Y^{*}$ is the bias due to ND a posteriori and $I_{R}^{*}$ is the associated ND uncertainty. With FY uncorrelated, the posterior bias is between $0.2 \%(\mathrm{MOX} 10 \mathrm{GWd} / \mathrm{t} ; 5000 \mathrm{~s})$ and $1.5 \%(\mathrm{MOX} 500 \mathrm{MWd} / \mathrm{t} ; 100 \mathrm{~s})$ and the posterior ND uncertainty is between 1.2 and $1.6 \%$, slightly reduced, as expected. With correlated FY, the conditions are slightly improved and the posterior bias is between 0.8 and $2.0 \%$ with a posterior uncertainty between 1.2 and $1.4 \%$. 
Table I. Transposition results - FY uncorrelated

\begin{tabular}{|ccc|c|c|c|c|cc|}
\hline \multicolumn{3}{|c|}{ Dickens fission burst exp. } & \multicolumn{6}{c|}{ MOX fuel } \\
\hline $\begin{array}{c}\text { E/C-1 } \\
{[\%]}\end{array}$ & $\begin{array}{c}\text { Exp. unc. } \\
{[\%]}\end{array}$ & $\begin{array}{c}\text { ND unc. } \\
{[\%]}\end{array}$ & $\begin{array}{c}\text { Burnup } \\
{[\mathrm{MWd} / \mathrm{t}]}\end{array}$ & $\begin{array}{c}\text { Cooling time } \\
{[\mathrm{s}]}\end{array}$ & $r_{R, E}$ & $I_{R}$ & $\delta Y^{*}$ & $I_{R}^{*}$ \\
{$[\%]$} & {$[\%]$} & {$[\%]$} \\
\hline 7.31 & 2.54 & 2.48 & & 100 & 0.68 & 1.49 & 1.48 & 1.31 \\
6.72 & 2.62 & 2.53 & & 200 & 0.70 & 1.52 & 1.38 & 1.33 \\
4.63 & 2.64 & 2.69 & 500 & 500 & 0.70 & 1.56 & 0.95 & 1.36 \\
4.58 & 2.99 & 3.02 & & 1000 & 0.69 & 1.59 & 0.84 & 1.39 \\
1.86 & 4.71 & 3.34 & & 2000 & 0.69 & 1.64 & 0.26 & 1.47 \\
1.86 & 4.71 & 3.34 & & 5000 & 0.66 & 1.76 & 0.27 & 1.60 \\
\hline 7.31 & 2.54 & 2.48 & & 100 & 0.67 & 1.48 & 1.45 & 1.31 \\
6.72 & 2.62 & 2.53 & & 200 & 0.69 & 1.51 & 1.35 & 1.32 \\
4.63 & 2.64 & 2.69 & & 500 & 0.69 & 1.55 & 0.92 & 1.35 \\
4.58 & 2.99 & 3.02 & 1000 & 1000 & 0.68 & 1.58 & 0.81 & 1.38 \\
1.86 & 4.71 & 3.34 & & 2000 & 0.67 & 1.63 & 0.25 & 1.47 \\
1.86 & 4.71 & 3.34 & & 5000 & 0.62 & 1.76 & 0.25 & 1.61 \\
\hline 6.71 & 2.55 & 2.36 & & 100 & 0.67 & 1.37 & 1.25 & 1.21 \\
7.31 & 2.54 & 2.48 & & 200 & 0.68 & 1.39 & 1.37 & 1.22 \\
6.14 & 2.46 & 2.58 & \multirow{2}{*}{10000} & 500 & 0.67 & 1.41 & 1.15 & 1.24 \\
4.72 & 2.72 & 2.81 & & 1000 & 0.65 & 1.44 & 0.79 & 1.28 \\
1.86 & 4.71 & 3.35 & & 2000 & 0.62 & 1.48 & 0.21 & 1.35 \\
1.86 & 4.71 & 3.35 & & 5000 & 0.56 & 1.59 & 0.21 & 1.48 \\
\hline
\end{tabular}

Table II. Transposition results - FY correlated

\begin{tabular}{|ccc|c|c|c|c|cc|}
\hline \multicolumn{3}{|c|}{ Dickens fission burst exp. } & \multicolumn{9}{c|}{ MOX fuel } \\
\hline $\begin{array}{c}\text { E/C-1 } \\
{[\%]}\end{array}$ & $\begin{array}{c}\text { Exp. unc. } \\
{[\%]}\end{array}$ & $\begin{array}{c}\text { ND unc. } \\
{[\%]}\end{array}$ & $\begin{array}{c}\text { Burnup } \\
{[\mathrm{MWd} / \mathrm{t}]}\end{array}$ & $\begin{array}{c}\text { Cooling time } \\
{[\mathrm{s}]}\end{array}$ & $r_{R, E}$ & $I_{R}$ & $\delta Y^{*}$ & $I_{R}^{*}$ \\
\hline 6.72 & 2.62 & 1.99 & & 100 & 0.82 & 1.63 & 1.96 & 1.37 \\
6.72 & 2.62 & 1.99 & & 200 & 0.83 & 1.61 & 1.94 & 1.35 \\
6.14 & 2.46 & 2.16 & & 500 & 0.83 & 1.62 & 1.78 & 1.34 \\
5.70 & 2.55 & 2.35 & 500 & 1000 & 0.84 & 1.67 & 1.62 & 1.36 \\
4.72 & 2.72 & 2.95 & & 2000 & 0.83 & 1.70 & 1.17 & 1.36 \\
4.72 & 2.72 & 2.95 & & 5000 & 0.77 & 1.56 & 1.01 & 1.30 \\
\hline 6.72 & 2.62 & 1.99 & & 100 & 0.82 & 1.59 & 1.90 & 1.34 \\
6.72 & 2.62 & 1.99 & & 200 & 0.82 & 1.56 & 1.86 & 1.31 \\
6.14 & 2.46 & 2.16 & \multirow{2}{*}{1000} & 500 & 0.82 & 1.57 & 1.71 & 1.30 \\
5.70 & 2.55 & 2.35 & & 1000 & 0.82 & 1.62 & 1.55 & 1.33 \\
4.63 & 2.64 & 2.62 & & 2000 & 0.83 & 1.64 & 1.20 & 1.33 \\
4.72 & 2.72 & 2.95 & & 5000 & 0.75 & 1.50 & 0.93 & 1.26 \\
\hline 6.72 & 2.62 & 1.99 & & 100 & 0.82 & 1.41 & 1.67 & 1.19 \\
6.72 & 2.62 & 1.99 & & 200 & 0.81 & 1.39 & 1.63 & 1.18 \\
6.14 & 2.46 & 2.16 & \multirow{2}{*}{10000} & 500 & 0.82 & 1.42 & 1.54 & 1.18 \\
5.70 & 2.55 & 2.35 & & 1000 & 0.81 & 1.43 & 1.35 & 1.18 \\
4.63 & 2.64 & 2.62 & & 2000 & 0.80 & 1.43 & 1.00 & 1.19 \\
4.72 & 2.72 & 2.95 & & 5000 & 0.72 & 1.30 & 0.78 & 1.11 \\
\hline
\end{tabular}




\section{CONCLUSIONS}

The decay heat is a crucial integral quantity for all fuels during a large time range, from reactor shutdown until geological times. Its computation involves more than 40,000 nuclear data and there are very few experiments available dedicated to its experimental validation and none for MOX fuels. Currently, the uncertainty quantification of the decay heat of MOX fuels mainly relies on ND covariance propagation through calculations. The resulting uncertainties depend on the cooling time and may be at a very low level, especially when taking into account FY correlations. This point is questioning and needs to be consolidated. The use of the transposition method, when it is possible, increases the control of the ND uncertainties and gives more consistency for nuclear safety authorities.

The results published in this paper do not lead to a strong uncertainty reduction for two reasons. First, FBE are associated with experimental uncertainties larger than ND uncertainties (2 to $10 \%$ at 1 std compared with 2 to $5 \%$ for ND uncertainties). Second, the representativity factor between an irradiated fuel and a FBE is limited to the part of the FY contribution to the total variance of the decay heat. Nevertheless, the use of FBE through the transposition process strengthens the uncertainty quantification based on ND covariance propagation for the decay heat at very short cooling times. Especially because it would be very difficult to design an integral experiment able to measure the total decay heat of an irradiated fuel before 20 minutes of cooling.

This study also points out several issues that need to be dealt with in the near future. First, the hypothesis that the computation bias on decay heat is only due to ND must be checked. Then, it should be interesting to transpose all the experimental points of the Dickens experiment jointly as experimental correlations are provided. This work could be completed with the other FBE of ${ }^{239} \mathrm{Pu}_{\text {th }}$ and the ones of ${ }^{241} \mathrm{Pu}_{\text {th }}$ and ${ }^{238} \mathrm{U}_{\text {fast }}$ in order to cover all the fission products contributions of a MOX fuel. The other sets of FBE data could be studied as well. The cross-section part of the decay heat uncertainty could still be dealt with covariance propagation. Indeed, the accuracy and the reliability of cross-section covariances are currently higher than for decay data or fission yields and are subjects to long term improvement programs worldwide.

Eventually, the need for new experiments must be emphasized, especially at short and intermediate cooling times. There is a crucial lack of experimental data for MOX fuels, but also for UOX fuels between the MERCI-1 experiment (45 minutes - 42 days) and other calorimetric measurements GE, HEDL, CLAB (2 years - 13 years), that cannot be filled with transposition methods only. It could be interesting also to design an experiment similar to FBE but with a depletion period long enough to deal with the accumulation of long-life fission products.

\section{REFERENCES}

[1] L. San Felice, R. Eschbach et P. Bourdot, «Experimental validation of the DARWIN2.3 package for fuel cycle applications,» Nuclear Technology, vol. 184, pp. 217-232, 2013.

[2] A. Santamarina et al., «The JEFF-3.1.1 nuclear data library,» JEFF Report 22, NEA/OECD, 2009.

[3] C. Fiche, «Mesure de la puissance résiduelle totale émise par les produits de fission thermique de 239Pu et 233U,» NEACRP/L, 1978.

[4] H. Nguyen, «Decay heat measurements following neutron fission of $235 \mathrm{U}$ and $239 \mathrm{Pu}, »$ International Conference on Nuclear Data for Science and Technology, Trieste, Italy, 1997.

[5] P. Johansson, «Integral determination of the beta and gamma heat in thermal neutron-induced fission of $235 \mathrm{U}$ and $239 \mathrm{Pu}$ and of the gamma heat in fast fission of $238 \mathrm{U}$,» Specialist's meeting (AEN/OCDE), Studsvik, Sweden, 1987.

[6] V. Vallet, C. Vaglio-Gaudard et C. Carmouze, «Application of the bias transposition method on 
PWR decay heat calculations with the DARWIN2.3 package,» GLOBAL2017, Seoul, Korea, 2017.

[7] J. Huyghe, V. Vallet, D. Lecarpentier, C. Reynard-Carette et C. Vaglio-Gaudard, «How to obtain an enhanced extended uncertainty associated with decay heat calculations of industrial PWRs using the DARWIN2.3 package,» EPJ Nuclear Sciences and Technology, vol. 5, 2019.

[8] J. Huyghe, C. De Saint Jean, D. Lecarpentier, C. Reynard-Carette, C. Vaglio-Gaudard et V. Vallet, «Integral data assimilation of the MERCI-1 experiment for the nuclear data associated with the PWR decay heat computation,» WONDER2018, Aix-en-Provence, France, 2018.

[9] A. Tsilanizara, "DARWIN: an Evolution Code System for a Large Range of Application," Journal of nucl. Sci. and Tech., vol. Sup. 1, pp. 845-849, 2003.

[10] V. Vallet, "Validation of the uncertainty propagation method for the decay heat within de DARWIN2.3 package,» BEPU 2018, Lucca, Italy, 2018.

[11] N. Terranova, O. Serot, P. Archier, C. De Saint Jean et M. Sumini, «Covariance matrix evaluations for independent mass fission yields,» Nuclear Data Sheets, vol. 123, pp. 225-230, 2015.

[12] K. H. Schmidt, B. Jurado, C. Amouroux et C. Schmitt, «General description of fission observables: GEF model code,» Nuclear Data Sheets, vol. 131, pp. 107-221, 2016.

[13] D. Rochman, O. Leray, A. Vasiliev, H. Ferroukhi, A. J. Koning, M. Fleming et J. C. Sublet, «A bayesian Monte Carlo method for fission yield covariance information,» Annals of Nuclear Energy, vol. 95, pp. 125-134, 2016.

[14] L. Fiorito, A. Stankovskiy, G. Van Den Eynde, C. Diez, O. Cabellos et P. E. Labeau, «Generation of fission yield covariances to correct discrepancies in the nuclear data libraries,» Annals of Nuclear Energy, vol. 88, pp. 12-23, 2016.

[15] P. Archier et al., «COMAC: nuclear data covariance matrice library for reactor applications,» PHYSOR 2014, Kyoto, Japan, 2014.

[16] A. Santamarina et D. Bernard, «Re-estimation of nuclear data and reliable covariances using integral experiments. Application to JEFF3 library.,» M\&C2017, Jeju, Korea, 2017.

[17] C. De Saint Jean, P. Archier, E. Privas, G. Noguere, O. Litaize et P. Leconte, «Evaluation of cross section uncertainties using physical constraints: focus on integral experiments,» Nuclear Data Sheets, vol. 123, pp. 225-230, 2015.

[18] J. Dickens, T. Love, J. MacConnel et R. Peelle, «Fission-product energy release for times following thermal-neutron fission of plutonium-239 and plutonium-241 between 2 and $14000 \mathrm{~s}, »$ Nuclear Science and Engineering, vol. 78, pp. 126-146, 1981.

[19] V. Orlov et al., «Problems of fast reactor physics related to breeding,» Energy Review, vol. 4, pp. 991-1075, 1980.

[20] P. Blaise, D. Bernard, C. De Saint Jean, N. Dos Santos, P. Leconte, A. Santamarina, J. Tommasi et J. F. Vidal, «The Uncertainty Quantification for complex neutronics code packages: issues on modern nuclear data assimilation and transposition to future nuclear systems,» chez ASME2011 PVP-MF, Baltimore, Maryland, USA, 2011.

[21] N. Dos Santos, P. Blaise et A. Santamarina, «A global approach of the representativity concept. Application on a high-conversion sub-moderated MOX lattice case,» M\&C2013, Sun Valley, Idaho, USA, 2013. 